\title{
BMJ Open Clinical characteristics, precipitating factors, management and outcome of patients with prior stroke hospitalised with heart failure: an observational report from the Middle East
}

Hadi A R Khafaji, ${ }^{1}$ Kadhim Sulaiman, ${ }^{2}$ Rajvir Singh, ${ }^{3}$ Khalid F AlHabib, ${ }^{4}$ Nidal Asaad, ${ }^{5}$ Alawi Alsheikh-Ali, ${ }^{6}$ Mohammed Al-Jarallah, ${ }^{7}$ Bassam Bulbanat, ${ }^{7}$ Wael AIMahmeed, ${ }^{5}$ Mustafa Ridha, ${ }^{8}$ Nooshin Bazargani, ${ }^{9}$ Haitham Amin, ${ }^{10}$ Ahmed Al-Motarreb, ${ }^{11}$ Hussam AlFaleh, ${ }^{4}$ Abdelfatah Elasfar, ${ }^{12,13}$ Prashanth Panduranga, ${ }^{2}$ Jassim Al Suwaidi ${ }^{3,14}$

To cite: Khafaji HAR, Sulaiman K, Singh $\mathrm{R}$, et al. Clinical characteristics, precipitating factors, management and outcome of patients with prior stroke hospitalised with heart failure: an observational report from the Middle East. BMJ Open 2015;5:e007148. doi:10.1136/bmjopen-2014007148

\section{- Prepublication for this} paper is available online. To view these files please visit the journal online (http://dx.doi.org/10.1136/ bmjopen-2014-007148).

Received 10 November 2014 Revised 20 February 2015 Accepted 1 March 2015

CrossMark

For numbered affiliations see end of article.

Correspondence to Dr Jassim Al Suwaidi; jalsuwaidi@hotmail.com

\section{ABSTRACT}

Objectives: The purpose of this study is to report the prevalence, clinical characteristics, precipitating factors, management and outcome of patients with prior stroke hospitalised with acute heart failure (HF).

Design: Retrospective analysis of prospectively collected data.

Setting: Data were derived from Gulf CARE (Gulf aCute heArt failuRe rEgistry), a prospective multicentre study of consecutive patients hospitalised with acute HF in 2012 in seven Middle Eastern countries and analysed according to the presence or absence of prior stroke; demographics, management and outcomes were compared.

Participants: A total of 5005 patients with HF.

Outcome measures: In-hospital and 1-year outcome. Results: The prevalence of prior stroke in patients with $\mathrm{HF}$ was $8.1 \%$. Patients with stroke with HF were more likely to be admitted under the care of internists rather than cardiologists. When compared with patients without stroke, patients with stroke were more likely to be older and to have diabetes mellitus, hypertension, atrial fibrillation, hyperlipidaemia, chronic kidney disease, ischaemic heart disease, peripheral arterial disease and left ventricular dysfunction ( $p=0.001$ for all). Patients with stroke were less likely to be smokers $(0.003)$. There were no significant differences in terms of precipitating risk factors for HF hospitalisation between the two groups. Patients with stroke with HF had a longer hospital stay (mean $\pm S D$ days; $11 \pm 14$ vs 9 $\pm 13, p=0.03$ ), higher risk of recurrent strokes and 1-year mortality rates $(32.7 \%$ vs $23.2 \%, p=0.001)$. Multivariate logistic regression analysis showed that stroke is an independent predictor of in-hospital and 1-year mortality.

Conclusions: This observational study reports high prevalence of prior stroke in patients hospitalised with $\mathrm{HF}$. Internists rather than cardiologists were the predominant caregivers in this high-risk group. Patients with stroke had higher risk of in-hospital recurrent strokes and long-term mortality rates.

\section{Strengths and limitations of this study}

- This is the first multicentre multinational study in the Middle East to provide a report on the prevalence, demographics, management and 1-year outcome of patients hospitalised with HF in relation to a history of stroke that included a relatively large number of patients.

- As an observational study, the possibility for unmeasured confounding biases exists. The current study has not recorded the cognitive status and the disability status in patients with stroke, which have a major impact on morbidity.

- Furthermore, no information is available regarding the cause of stroke (embolic vs thrombotic), and future studies need to overcome these limitations.

- Mortality rates at follow-up were only recorded at 1 year without the specification of the exact date of death of each patient.

Trial registration number: NCT01467973.

\section{INTRODUCTION}

The prevalence and incidence of stroke in patients with heart failure (HF) and the temporal relation of these two diseases in terms of increasing morbidity and mortality have scarcely been reported from around the world and never from the Middle East. HF is one of the leading causes of hospitalisation, and is a significant factor for morbidity and mortality worldwide. Moreover, HF is a major risk factor for the development of ischaemic stroke with a 2-3-fold increased risk of stroke when compared with patients without HF. ${ }^{1}$ Factors that 
may contribute to the occurrence of stroke in HF include: atrial fibrillation (AF) or left ventricular (LV) dysfunction that are potential source embolisation. ${ }^{2} 3$ The hypercoagulable state and reduced fibrinolysis are a consequence of the activation of the sympathetic nervous system and the reninangiotensin-aldosterone system. ${ }^{4}$ In addition to endothelial dysfunction in $\mathrm{HF}^{6}{ }^{7}$ hypotension may also be an additional risk factor for stroke as a result of $\mathrm{HF}^{8}$ Moreover, underlying risk factors for the development of HF, such as hypertension and diabetes mellitus, ${ }^{9}$ can also predispose to large-artery atherosclerosis and small-vessel thrombosis and hence stroke. $^{10}$

The prevalence and incidence of prior and acute stroke in patients with HF is unclear because of the heterogeneous nature of the limited published studies, most of which were subset analyses of randomised trials rather than epidemiological studies. ${ }^{11-17}$ Furthermore, most of the available data are mainly limited to studies conducted in the western world and data about the prevalence of prior stroke among patients hospitalised with $\mathrm{HF}$ are lacking. The aim of this study is to define the prevalence, clinical characteristics, precipitating factors, management and outcome of patients with stroke hospitalised with HF, using data from Gulf CARE (Gulf aCute heArt failuRe rEgistry). ${ }^{18}$ It is hypothesised that patients with prior stroke when hospitalised with HF have worse outcomes when compared with patients with $\mathrm{HF}$ and without prior stroke.

\section{PATIENTS AND METHODS}

\section{Registry design}

Gulf CARE is a prospective multinational multicentre registry that recruited patients from February 2012 to November 2012 who were admitted with the final diagnosis of acute HF (AHF) in 47 hospitals in seven Middle Eastern Arab countries in the Gulf. ${ }^{18}$ Data were collected on episodes of hospitalisation beginning with point of initial care, with patient's discharge, transfer out of hospital or in-hospital death, and for those discharged alive 3 and 12 months follow-up was obtained. Patients' recruitment was preceded by a pilot phase of 1 month in November 2011. Institutional or national ethical committee or review board approval was obtained in each of the seven participating countries, and all patients provided informed consent. Each patient was given a unique identification number to prevent double counting. The study is registered at clinicaltrials.gov with the number NCT01467973.

Patients included in this registry were men and women aged 18 years and over with AHF who were admitted to participating hospitals. AHF was defined based on the European Society of Cardiology (ESC) definition ${ }^{2}$ and was further classified as either acute decompensated chronic HF (ADCHF) or new-onset AHF (de novo AHF) based on the ESC guidelines. ADCHF was defined as worsening of HF in patients with a previous diagnosis or hospitalisation for HF. New-onset AHF (de novo AHF) was defined as AHF in patients with no history of HF.
Patients were excluded from Gulf CARE if: (1) they were discharged from the emergency room without admission. (2) They were transferred from a non-registry hospital. (3) There was a failure to obtain informed consent. (4) Their final diagnosis was not HF, in which case they were also excluded from the final analyses. Registry organisation and Data Collection and Validation are mentioned in Gulf CARE. ${ }^{18}$

Definitions of data variables in the chronic renal failure (CRF) are based on the ESC guidelines 2008 and the American College of Cardiology clinical data standards 2005. ${ }^{19}{ }^{20}$ Cardiomyopathy was defined as a myocardial disorder in which the heart muscle is structurally and functionally abnormal (in the absence of coronary artery disease, hypertension, valvular disease or congenital heart disease) sufficient to cause the observed myocardial abnormality. ${ }^{20}$ Diastolic HF was defined as the presence of symptoms and/or signs of $\mathrm{HF}$ and a preserved LV ejection fraction (LVEF) $>40 \% .{ }^{19}$ Stroke/transient ischaemic attack (TIA) was defined as a history of cerebrovascular disease, documented by any one of the following: (1) cerebrovascular ischaemic or haemorrhagic stroke: patient has a history of stroke (ie, any focal neurological deficit of abrupt onset caused by a disturbance in blood supply that did not resolve within $24 \mathrm{~h}$ ) confirmed by a standard neurological examination with or without a positive imaging study, or an event of presumed ischaemic origin that did not resolve within $24 \mathrm{~h}$, but the imaging showed a new lesion. (2) TIA: patient has a history of any sudden new focal neurological deficit of presumed ischaemic origin as determined by a standard neurological examination that resolved completely within $24 \mathrm{~h}$, with a brain image study not revealing a new lesion. (3) Non-invasive/invasive carotid test with occlusion greater than or equal to $75 \%$. (4) Previous carotid artery surgery. (5) Previous carotid angioplasty. ${ }^{19}$ Diabetes mellitus was defined as having a history of diabetes diagnosed and treated with medication and/or insulin or fasting blood glucose $7.0 \mathrm{mmol} / \mathrm{L}(126 \mathrm{mg} / \mathrm{dL})$ or glycated haemoglobin (HbAlc) $\geq 6.5 \%$. Hypertension was defined as having a history of hypertension diagnosed and treated with medication, blood pressure greater than $140 \mathrm{~mm} \mathrm{Hg}$ systolic or $90 \mathrm{~mm} \mathrm{Hg}$ diastolic on at least two occasions or greater than $130 \mathrm{~mm} \mathrm{Hg}$ systolic or $80 \mathrm{~mm} \mathrm{Hg}$ diastolic on at least two occasions for patients with diabetes or chronic kidney disease (CKD). Hyperlipidaemia was defined as a history of dyslipidemia diagnosed and/or treated by a physician or total cholesterol greater than 5.18 $\mathrm{mmol} / \mathrm{L}$ (200 mg/dL), low-density lipoprotein greater than or equal to $3.37 \mathrm{mmol} / \mathrm{L}(130 \mathrm{mg} / \mathrm{dL})$ or highdensity lipoprotein less than $1.04 \mathrm{mmol} / \mathrm{L}(40 \mathrm{mg} / \mathrm{dL})$. Current smoker was defined as a person smoking cigarettes, a water pipe, a cigar or chewing tobacco within 1 month of index admission.

CKD was defined as glomerular filtration rate (GFR) $<60 \mathrm{~mL} / \mathrm{min} / 1.73 \mathrm{~m}^{2}$ for 3 months or more, with or without kidney damage or on dialysis. If no GFR was available, serum creatinine $>177 \mathrm{mmol} / \mathrm{L}$ or $2 \mathrm{mg} / \mathrm{dL}$ was marked as CKD. Obesity was defined as body mass 
index greater than $25 \mathrm{~kg} / \mathrm{m}^{2}$. In the registry, infection is defined as any systemic infection needing antibiotics. ${ }^{20}$

\section{Statistical analysis}

Baseline and outcome data are presented in frequency and percentages for categorical variables and interval variables are presented in mean and SDs or median and range as appropriate. In order to establish the association between stroke and non-stroke groups $\chi^{2}$ tests were applied. Student t tests or Wilcoxon rank sum tests were used for interval variables between the two groups as appropriate. Multivariate logistic regression analysis was performed at in-hospital and 1 year mortality for important risk factors. Adjusted OR and $95 \%$ CI with $\mathrm{p}$ values are presented in table 6. $p$ Value $\leq 0.05$ (two tailed) is considered statistically significant. SPSS V.21.0 Statistical package was used for the analysis. ${ }^{21}$

\section{RESULTS}

\section{Patient demographics}

Approximately $8.1 \%$ of Gulf CARE patients (total 5005 patients) had a history of stroke, with no significant gender or racial differences between stroke and nonstroke groups (tables 1-3). Patients with stroke with HF were older ( 66.5 vs 59 years, $\mathrm{p}=0.001$ ) and more likely to have diabetes mellitus, hypertension, $\mathrm{AF}$, coronary artery disease and LV dysfunction $(\mathrm{p}=0.001)$. Patients with stroke were also more likely to have chronic kidney disease and to be on renal replacement therapy than patients without stroke $(\mathrm{p}=0.001)$ and to have thyroid disease $(6.2 \%$ vs $3.4 \%, \mathrm{p}=0.001)$ and previous coronary artery bypass grafting ( $7 \%$ vs $10.9 \%, \mathrm{p}=0.004$ ) and less likely to be smokers $(p=0.003)$ or have a history of asthma/chronic obstructive pulmonary disease. Patients with stroke were more likely to present with ADCHF

$\underline{\text { Table } 1 \text { Baseline demographic and clinical characteristics (no stroke/TIA vs stroke/TIA) }}$

\begin{tabular}{|c|c|c|c|}
\hline Variable & $\begin{array}{l}\text { No stroke/TIA } \\
\mathrm{N}=4601(91.9 \%)\end{array}$ & $\begin{array}{l}\text { Stroke/TIA } \\
\mathrm{N}=404(8.1 \%)\end{array}$ & p Value \\
\hline Acute new-onset heart failure (\%) & $2150(46.7)$ & $126(31.2)$ & \\
\hline Acute decompensated chronic heart failure (\%) & 2451 (53.3) & $278(68.8)$ & 0.001 \\
\hline Age (mean $\pm \mathrm{SD})$ & $59 \pm 14.9$ & $66.5 \pm 13$ & 0.001 \\
\hline \multicolumn{4}{|l|}{ Gender } \\
\hline Male (\%) & 2892 (62.9) & $239(59.2)$ & \\
\hline Female (\%) & 1709 (31.1) & $165(40.8)$ & 0.14 \\
\hline \multicolumn{4}{|l|}{ Ethnicity } \\
\hline Arab (\%) & $4130(89.8)$ & $386(95.5)$ & \\
\hline Asians (\%) & $455(9.9)$ & $18(4.5)$ & \\
\hline Others (\%) & $16(0.3)$ & $0(0)$ & 0.001 \\
\hline \multicolumn{4}{|l|}{ Main caregiver } \\
\hline Cardiologist (\%) & 3326 (72.3) & $249(61.6)$ & \\
\hline Internist (\%) & $1275(27.7)$ & $155(38.4)$ & 0.001 \\
\hline \multicolumn{4}{|l|}{ Previous CV history } \\
\hline \multicolumn{4}{|l|}{ HF previous admission (\%) } \\
\hline$\leq 6$ months $(\%)$ & $2439(53)$ & $278(68.8)$ & 0.001 \\
\hline Known systolic LV dysfunction (\%) & $2053(44.6)$ & $228(56.4)$ & 0.001 \\
\hline Known CAD (\%) & $2083(45.3)$ & 254 (62.9) & 0.001 \\
\hline Valvular heart disease (\%) & 608 (13.2) & 67 (16.6) & 0.06 \\
\hline PVD (\%) & $162(3.5)$ & $61(15.1)$ & 0.001 \\
\hline Atrial fibrillation (\%) & $569(11.1)$ & $98(24.3)$ & 0.001 \\
\hline Current smoking (\%) & $1038(22.6)$ & $65(16.1)$ & 0.003 \\
\hline Type 1 DM (\%) & $160(3.5)$ & $25(6.2)$ & \\
\hline Type 2 DM (\%) & $2052(44.6)$ & $255(63.1)$ & 0.001 \\
\hline Known HTN (\%) & $2718(59.1)$ & 341 (84.4) & 0.001 \\
\hline Known hyperlipidaemia (\%) & 1572 (34.2) & $227(56.2)$ & 0.001 \\
\hline CKD/dialysis (\%) & $631(13.7)$ & $113(28)$ & 0.001 \\
\hline Sleep apnoea requiring therapy (\%) & $88(1.9)$ & $11(2.7)$ & 0.26 \\
\hline Family history of cardiomyopathy/heart failure (\%) & $244(5.3)$ & $15(3.7)$ & 0.17 \\
\hline Khat $(\%)$ & $852(18.5)$ & $39(9.7)$ & 0.001 \\
\hline Alcohol (\%) & $165(3.6)$ & $11(2.7)$ & 0.63 \\
\hline Peripartum (at present) (\%) & $76(1.7)$ & $2(0.5)$ & 0.07 \\
\hline Radiation (\%) & $19(0.4)$ & $0(0)$ & 0.20 \\
\hline Chemotherapy (\%) & $29(0.6)$ & $3(0.7)$ & 0.79 \\
\hline Thyroid disease (\%) & $156(3.4)$ & $25(6.2)$ & 0.001 \\
\hline Asthma/COPD (\%) & $446(9.7)$ & $55(13.6)$ & 0.01 \\
\hline
\end{tabular}


Table 2 Clinical presentation (symptoms, signs; stroke/TIA vs no stroke/TIA)

\begin{tabular}{|c|c|c|c|}
\hline Variable & $\begin{array}{l}\text { No stroke/TIA } \\
\mathrm{N}=4601 \text { (91.9\%) }\end{array}$ & $\begin{array}{l}\text { Stroke/TIA, } \\
\mathrm{N}=404(8.1 \%)\end{array}$ & p Value \\
\hline \multicolumn{4}{|l|}{ Clinical symptoms } \\
\hline Cardiac arrest (\%) & $138(3)$ & $19(4.7)$ & 0.06 \\
\hline NYHA I (\%) & $124(2.7)$ & $5(1.2)$ & \\
\hline NYHA II (\%) & $933(20.3)$ & $72(17.8)$ & \\
\hline NYHA III (\%) & $1973(42.9)$ & $188(46.5)$ & \\
\hline NYHA IV (\%) & $1471(32)$ & $132(32.7)$ & 0.22 \\
\hline Orthopnoea (\%) & $3618(78.6)$ & $324(80.2)$ & 0.46 \\
\hline Paroxysmal nocturnal dyspnoea (\%) & 2942 (63.9) & $274(67.8)$ & 0.12 \\
\hline Abdominal/lower limb swelling (\%) & $2055(44.7)$ & $187(46.3)$ & 0.53 \\
\hline Weight gain (yes) (\%) & $1207(26.2)$ & $100(24.8)$ & 0.52 \\
\hline Chest pain (\%) & 2034 (44.2) & $166(41.1)$ & 0.23 \\
\hline Palpitation (\%) & $1413(30.7)$ & $107(26.5)$ & 0.08 \\
\hline Easy fatigability (\%) & 2604 (56.6) & $230(56.9)$ & 0.89 \\
\hline Syncope in the last 1 year (\%) & $218(4.7)$ & 45 (11.1) & 0.001 \\
\hline \multicolumn{4}{|l|}{ Clinical signs } \\
\hline Heart rate $($ mean $\pm S D)$ & $97 \pm 23$ & $95 \pm 22.9$ & 0 \\
\hline \multicolumn{4}{|l|}{$\mathrm{BP}(\mathrm{mm} \mathrm{Hg})($ mean $\pm \mathrm{SD})$} \\
\hline Systolic & $137 \pm 34$ & $142 \pm 33$ & 0.01 \\
\hline Diastolic & $81 \pm 20$ & $80 \pm 19.5$ & 0.37 \\
\hline $\mathrm{RR}(\operatorname{mean} \pm \mathrm{SD})$ & $24.6 \pm 5.9$ & $24.9 \pm 5.8$ & 0.32 \\
\hline Weight $(\mathrm{kg})($ mean $\pm \mathrm{SD})$ & $74 \pm 17$ & $76 \pm 17.6$ & 0.02 \\
\hline Height $(\mathrm{cm})($ mean $\pm S D)$ & $162 \pm 8.6$ & $163 \pm 9$ & 0.56 \\
\hline Waist circumference $(\mathrm{cm})($ mean $\pm S D)$ & $92 \pm 15$ & $93 \pm 16.7$ & 0.84 \\
\hline $\mathrm{BMI}\left(\mathrm{kg} / \mathrm{m}^{2}\right)($ mean $\pm \mathrm{SD})$ & $28 \pm 6$ & $29 \pm 6.3$ & 0.03 \\
\hline Raised JVP>6 cm (\%) & $2323(56.5)$ & $203(50.2)$ & 0.93 \\
\hline Peripheral oedema (\%) & $2496(54.2)$ & $231(57.2)$ & 0.26 \\
\hline Ascites (\%) & $658(14.3)$ & $65(16.1)$ & 0.33 \\
\hline Enlarged tender liver (\%) & $1260(27.4)$ & 78 (19.3) & 0.001 \\
\hline Gallop (\%) & $1747(38)$ & $129(31.9)$ & 0.02 \\
\hline Basal lung crepitation (\%) & $4214(91.6)$ & $383(94.8)$ & 0.02 \\
\hline Signs of pleural effusion (\%) & $847(18.4)$ & $77(19.1)$ & 0.75 \\
\hline
\end{tabular}

with more frequent recent ( $\leq 6$ months) HF hospitalisations $(p=0.001)$, and more likely to be admitted under the care of internists rather than cardiologists when compared with patients without stroke.

\section{Investigations during hospitalisation}

Patients with stroke were more likely to be admitted with $\mathrm{AF}$ when compared with patients without stroke; atrial fibrillation/flutter ( $12.7 \%$ vs $24.9 \%, \mathrm{p}=0.001$; table 4 ). Patients with stroke were also more likely to have concentric LV hypertrophy ( $26.8 \%$ vs $32.7 \%, \mathrm{p}=0.02$ ), and less likely to have mitral regurgitation $(30.4 \%$ vs $22.5 \%$, $\mathrm{p}=0.001$ ) with no differences in the mean EF on echocardiographic assessment between the two groups.

Patients with stroke were more likely to have a lower GFR $($ mean $\pm S D ; 58 \pm 36.6$ vs $69 \pm 35.7, \mathrm{p}=0.001$ and, as a result, higher serum creatinine $($ mean \pm SD; $146 \pm 111$ vs $129 \pm 117 \mathrm{mg} / \mathrm{dL}, \mathrm{p}=0.003$ ) and blood urea (mean $\pm \mathrm{SD}$ : $12.8 \pm 9$ vs $11 \pm 8.4 \mathrm{mg} / \mathrm{dL}, \mathrm{p}=0.002$ ). Patients with stroke were also more likely to have lower admission haemoglobin levels (mean $\pm \mathrm{SD} ; 11.9 \pm 2.3$ vs $12.7 \pm 2.4 \mathrm{mg} / \mathrm{dL}$, $\mathrm{p}=0.001$ ).
Precipitating factors, hospitalisation course and outcomes There were no significant differences in terms of precipitating factors for HF hospitalisation between the two groups (tables 5 and 6 ).

Patients with stroke were more likely to require invasive and non-invasive ventilations $(12.4 \%$ vs $8.1 \%$, $\mathrm{p}=0.003)$, $(15.3 \%$ vs $8.9 \%, \mathrm{p}=0.001)$, respectively, and were also more likely to require inotropic support (21.8\% vs $15.1 \%, \mathrm{p}=0.001)$, AF therapy $(11.4 \%$ vs $5.8 \%$, $\mathrm{p}=0.001 \%)$, renal replacement therapy $(2.5 \%$ vs $4.5 \%$, $\mathrm{p}=0.02$ ) and blood transfusions (9.2\% vs $4.7 \%, \mathrm{p}=0.001$ ) when compared with patients without stroke. Patients with stroke were also more likely to suffer recurrent strokes and have systemic infections that require antibiotic treatment $(34.9 \%$ vs $23.2 \%, p=0.001)$ during the same hospitalisation when compared with patients without stroke (table 5). The clinical workup of patients with stroke showed that they were more likely to have ischaemic heart disease $(59.2 \%$ vs $42.7 \%, \mathrm{p}=0.01)$ and less likely to have other types of cardiomyopathies ( $13.1 \%$ vs $18.7 \% \mathrm{p}=0.005)$, including idiopathic cardiomyopathy $(9.2 \%$ vs $13.1 \%, \mathrm{p}=0.02$; table 5$)$. 
Table 3 Medications and interventions (stroke/TIA vs no stroke/TIA)

\begin{tabular}{|c|c|c|c|c|c|c|}
\hline \multirow[b]{2}{*}{ Variable } & \multicolumn{3}{|l|}{ Before admission } & \multicolumn{3}{|l|}{ On discharge } \\
\hline & $\begin{array}{l}\text { No stroke/TIA } \\
\mathrm{N}=4601 \text { (91.9\%) }\end{array}$ & $\begin{array}{l}\text { Stroke/TIA } \\
\mathrm{N}=404(8.1 \%)\end{array}$ & p Value & $\begin{array}{l}\text { No stroke/TIA } \\
\mathrm{N}=4601(91.9 \%)\end{array}$ & $\begin{array}{l}\text { Stroke/TIA } \\
\mathrm{N}=404(8.1 \%)\end{array}$ & p Value \\
\hline Digoxin & $758(16.5)$ & $92(22.8)$ & 0.001 & $1112(24.2)$ & $95(23.5)$ & 0.77 \\
\hline Oral nitrates & $1151(25)$ & $154(38.1)$ & 0.001 & $1636(35.6)$ & $186(46)$ & 0.001 \\
\hline Hydralazine & $187(4.1)$ & $34(8.4)$ & 0.001 & $299(6.5)$ & $54(13.4$ & 0.001 \\
\hline Aspirin & $2781(60.4)$ & $308(76.2)$ & 0.001 & 3538 (76.9) & $313(77.5)$ & 0.80 \\
\hline Clopidogrel & 849 (18.5) & $117(29)$ & 0.001 & $1631(35.5)$ & 167 (41.3) & 0.02 \\
\hline Oral anticoagulants (\%) & $537(11.7)$ & $81(20)$ & 0.001 & $806(17.5)$ & $91(22.5)$ & 0.01 \\
\hline Statin & 2269 (49.3) & $286(70.8)$ & 0.001 & $311(67.7)$ & 319 (79) & 0.001 \\
\hline Allopurinol & $121(2.6)$ & $14(3.5)$ & 0.32 & $219(4.8)$ & $20(5)$ & 0.86 \\
\hline Ivabradine (\%) & 107 (2.3) & $8(2.0)$ & 0.66 & 227 (4.9) & $17(4.2)$ & 0.52 \\
\hline Antiarrhythmic (\%) & $116(2.5)$ & $10(2.5)$ & 0.96 & $208(4.5)$ & $15(3.7)$ & 0.45 \\
\hline Antidepressants (\%) & 57 (1.2) & $9(2.2)$ & 0.10 & $82(1.8)$ & $10(2.5)$ & 0.32 \\
\hline \multicolumn{7}{|l|}{ BB } \\
\hline Carvedilol & $992(49.6)$ & $105(50.2)$ & 0.87 & $1600(51.6)$ & $140(53.8)$ & 0.48 \\
\hline Metoprolol & $197(9.9)$ & $26(12.4)$ & 0.24 & $295(9.5)$ & 32 (12.3) & 0.14 \\
\hline Bisoprolol & $648(32.4)$ & $59(28.2)$ & 0.22 & $1151(37.1)$ & $80(30.8)$ & 0.04 \\
\hline ACE inhibitors & $1968(42.8)$ & $164(40.6)$ & 0.40 & 2694 (58.6) & $188(46.5)$ & 0.001 \\
\hline ARBs & $563(12.2)$ & $84(20.8)$ & 0.001 & 725 (15.8) & 77 (19.1) & 0.08 \\
\hline Aldosterone antagonists & 778 (16.9) & $62(15.3)$ & 0.42 & $1921(41.8)$ & $135(33.4)$ & 0.001 \\
\hline \multicolumn{7}{|l|}{ Cardiac procedures } \\
\hline $\mathrm{PCl}$ & $484(10.5)$ & $55(13.6)$ & 0.05 & $2861(6.2)$ & 13 (3.2) & 0.02 \\
\hline CABG & $322(7)$ & 44 (10.9) & 0.004 & $65(1.4)$ & $4(1.0)$ & 0.49 \\
\hline \multicolumn{7}{|l|}{ Device therapy (yes) (\%) } \\
\hline CRT-P & $4(0.1)$ & $1(0.2)$ & & $2(0)$ & $0(0)$ & \\
\hline CRT-D & $52(1.1)$ & $4(1.0)$ & & $26(0.6)$ & $3(0.7)$ & \\
\hline ICD & $80(1.7)$ & $12(3)$ & 0.37 & $45(1.0)$ & $8(2.0)$ & 0.10 \\
\hline Valve repair/replacement (yes) (\%) & $148(3.2)$ & $19(4.7)$ & 0.11 & $88(1.9)$ & $6(1.5)$ & 0.54 \\
\hline
\end{tabular}

Patients with stroke were more likely to be discharged on oral nitrates, hydralazine, statin, ACE inhibitors (ACEIs), aldosterone antagonists $(\mathrm{p}=0.001)$, oral anticoagulants $(p=0.02)$ and clopidogrel $(p=0.01)$ when compared with patients without stroke (table 3). In comparison with stroke patients non-stroke patients had percutaneous coronary intervention during hospitalisation $(\mathrm{p}=0.02)$.

Patients with stroke had a longer hospital stay (mean \pm SD days; $11 \pm 14$ vs $9 \pm 13, \mathrm{p}=0.03$ ) as well as a higher but statistically non-significant in-hospital $(8.4 \%$ vs $6.1 \%$, $\mathrm{p}=0.06$ ) and a significantly higher 1-year mortality rate $(32.7 \%$ vs $23.2 \%, \mathrm{p}=0.001$; table 5$)$. On multivariate logistic regression analysis, stroke was an independent mortality predictor for in-hospital and 1-year follow-up (table 6). Age, hypertension, peripheral vascular disease and AF were independent risk factors for stroke in patients with HF (table 6).

\section{Cardiologist vs internist care}

Subanalysis of patients with prior stroke according to whether the primary care provider is a cardiologist versus internist showed the following: patients admitted to cardiologists were more likely to be prescribed clopidogrel (48.2\% vs 30.3\%, p=0.001) and ACEI (51.8 vs
$38.1 \%, \mathrm{p}=0.007)$ and with less incidence of recurrent stroke ( $2 \%$ vs $11 \%, \mathrm{p}=0.001)$, less major bleeding $(0 \%$ vs $1.9 \%, \mathrm{p}=0.03)$ and the need for blood transfusion $(6.4 \%$ vs $13.5 \%, \mathrm{p}=0.02)$ when compared with patients with prior stroke hospitalised under the care of internists. Patients with prior stroke with systemic infection requiring antibiotics were more likely to be admitted under internal medicine care $(46.5 \%$ vs $27.7 \%, \mathrm{p}=0.001)$ with higher in-hospital mortality $(13.5 \%$ vs $5.2 \%$, $\mathrm{p}=0.009$ ) with no significant differences in the duration of hospital stay $(11 \pm 17$ vs $10 \pm 10$ (days), $p=0.33$ ) when compared with those admitted under cardiologist care. One year follow-up showed non-significant marginally higher rehospitalisation for patients under internist care $(19.3 \%$ vs $24.5 \%, \mathrm{p}=0.07)$ with significantly higher mortality when compared with patients under cardiologist care $(69.9 \%$ vs $63.2 \%$, $\mathrm{p}=0.002$; table 7 ).

\section{DISCUSSION}

The current registry of patients hospitalised for $\mathrm{HF}$ demonstrates the following: (1) stroke history is relatively common among Middle Eastern patients. (2) Patients with stroke had a higher risk of recurrent in-hospital 
Table 4 Investigations during hospitalisation (stroke/TIA vs no stroke/TIA)

\begin{tabular}{|c|c|c|c|}
\hline Variable & $\begin{array}{l}\text { No stroke/TIA } \\
\mathrm{N}=4601(91.9 \%)\end{array}$ & $\begin{array}{l}\text { Stroke/TIA } \\
\mathrm{N}=404(8.1 \%)\end{array}$ & p Value \\
\hline Pulse oximetry saturation (mean $\pm S D$ ) & $93 \pm 6.9$ & $93 \pm 6.2$ & 0.86 \\
\hline \multicolumn{4}{|l|}{ Troponin, $\mathrm{ng} / \mathrm{mL}$} \\
\hline Elevated (\%) & 1726 (37.5) & $176(43.6)$ & \\
\hline Normal (\%) & 2453 (53.3) & $198(49)$ & \\
\hline Not performed (\%) & $422(9.2)$ & $30(7.4)$ & 0.05 \\
\hline First BNP or NT-Pro BNP, pg/mL & $780(17)$ & $70(17.3)$ & 0.85 \\
\hline HbA1c (mean \pm SD) (\%) & $7.2 \pm 2.2$ & $7.6 \pm 2.0$ & 0.03 \\
\hline Total cholesterol $($ mean $\pm \mathrm{SD}) \mathrm{mmol} / \mathrm{L}$ & $4.8 \pm 2.3$ & $4.5 \pm 2.2$ & 0.02 \\
\hline First haemoglobin (mean $\pm S D$ ) g/dL & $12.7 \pm 2.4$ & $11.9 \pm 2.3$ & 0.001 \\
\hline First WCC (mean \pm SD) cells $/ \mathrm{mL}$ & $10.4 \pm 8$ & $10 \pm 7$ & 0.89 \\
\hline First urea $($ mean $\pm S D) ~ m m o l / L$ & $11 \pm 8.4$ & $12.8 \pm 9$ & 0.002 \\
\hline First creatinine (mean $\pm S D$ ) $\mathrm{mmol} / \mathrm{L}$ & $129 \pm 117$ & $146 \pm 111$ & 0.003 \\
\hline E-GFR $(\operatorname{mean} \pm S D) \mathrm{mL} / \mathrm{min}$ & $69 \pm 35.7$ & $58 \pm 36.6$ & 0.001 \\
\hline First serum potassium (mean $\pm \mathrm{SD}$ ) $\mathrm{mmol} / \mathrm{L}$ & $4.3 \pm 0.7$ & $4.3 \pm 0.69$ & 0.06 \\
\hline First ALT (mean $\pm S D) \mathrm{mmol} / \mathrm{L}$ & $94 \pm 218$ & $71 \pm 192$ & 0.02 \\
\hline \multicolumn{4}{|l|}{ ECG rhythm } \\
\hline Sinus (\%) & 3803 (83.2) & $288(71.8)$ & 0.01 \\
\hline $\mathrm{AF} /$ flutter (\%) & $579(12.7)$ & $100(24.9)$ & 0.001 \\
\hline Paced (\%) & $69(1.5)$ & $10(2.5)$ & 0.13 \\
\hline Others (\%) & $62(1.4)$ & $1(0.2)$ & 0.05 \\
\hline LVH (\%) & 1377 (29.9) & $144(35.6)$ & 0.02 \\
\hline STEMI (\%) & $495(10.8)$ & $31(7.7)$ & 0.05 \\
\hline $\mathrm{AF}$ & $509(11.1)$ & $98(24.3)$ & 0.001 \\
\hline $\mathrm{CHB}$ & $56(1.2)$ & $2(0.5)$ & 0.20 \\
\hline Pathological Q waves (old Ml) & $1072(23.3)$ & $106(16.2)$ & 0.18 \\
\hline QRS duration $\geq 0.12 \mathrm{~ms}$ & - & & \\
\hline LBBB & $596(13)$ & $61(15.1)$ & 0.22 \\
\hline RBBB & $203(4.4)$ & $19(4.7)$ & 0.79 \\
\hline IVCD & $142(3.1)$ & $17(4.2)$ & 0.22 \\
\hline Echocardiography (available) (\%) & 4207 (91.4) & $370(91.6)$ & 0.92 \\
\hline Left atrial enlargement (yes) (\%) & 2658 (63.2) & $239(64.6)$ & 0.59 \\
\hline LVEF (mean \pm SD) & $37 \pm 14$ & $36.5 \pm 13$ & 0.57 \\
\hline$E F \leq 35 \%$ & 1847 (43.9) & $165(44.6)$ & 0.80 \\
\hline$E F>35 \%$ & $2360(56.1)$ & $205(55.4)$ & 0.80 \\
\hline Concentric LVH & $1129(26.8)$ & $121(32.7)$ & 0.02 \\
\hline \multicolumn{4}{|l|}{ Moderate-to-severe valve disease } \\
\hline MS & $127(2.8)$ & $8(2)$ & 0.35 \\
\hline MR & $1400(30.4)$ & $91(22.5)$ & 0.001 \\
\hline AS & $115(2.5)$ & $8(2.0)$ & 0.52 \\
\hline AR & $177(3.8)$ & $12(3.0)$ & 0.38 \\
\hline TR & $646(14)$ & 49 (12.1) & 0.29 \\
\hline PA systolic pressure $($ mean $\pm S D)$ & $55.7 \pm 16$ & $53 \pm 11$ & 0.03 \\
\hline Coronary angiogram (within 1 year) (\%) & $1017(22.1)$ & 74 (18.3) & 0.08 \\
\hline SVD & $183(4)$ & $10(2.5)$ & 0.13 \\
\hline DVD & $204(4.4)$ & $10(2.5)$ & 0.06 \\
\hline TVD & $313(6.8)$ & $30(7.4)$ & 0.64 \\
\hline LMSD & $16(0.3)$ & $0(0)$ & 0.24 \\
\hline Blocked stent/graft & $23(0.5)$ & $6(1.5)$ & 0.01 \\
\hline \multicolumn{4}{|c|}{$\begin{array}{l}\text { AF, atrial fibrillation; ALT, alanine transaminase; AR, aortic regurgitation; AS, aortic stenosis; BNP, brain natriuretic peptide; CHB, complete } \\
\text { heart block; DVD, double vessel disease; EF, ejection fraction; GFR, glomerular filtration rate; HbA1c, glycated haemoglobin; IVCD, intra- } \\
\text { ventricular conduction delay; LBBB, left bundle branch block; LMSD, left main disease; LVH, left ventricular hypertrophy; LVEF, left ventricular } \\
\text { ejection fraction; MI, myocardial infarction; MR, mitral regurgitation; MS, mitral stenosis; NT-Pro BNP, N-terminal Pro brain natriuretic peptide; } \\
\text { PA, pulmonary artery; RBBB, right bundle branch block; STEMI, ST elevation myocardial infarction; SVD, single vessel disease; TIA, transient } \\
\text { ischaemic attack; TR, tricuspid regurgitation; TVD, tripe vessel disease, WCC, white cell count. }\end{array}$} \\
\hline
\end{tabular}

strokes and higher long-term mortality rates. (3) Prior stroke was an independent predictor of in-hospital and 1-year mortality. (4) Patients with stroke hospitalised with $\mathrm{HF}$ were more likely to be admitted under internist care rather than cardiologist care with less use of evidence-based medications. 
Table 5 Course in the hospital and in-hospital and 1-year outcome (stroke/TIA vs no stroke/TIA)

\begin{tabular}{|c|c|c|c|}
\hline Variable & $\begin{array}{l}\text { No stroke/TIA } \\
\mathrm{N}=4601(91.9 \%)\end{array}$ & $\begin{array}{l}\text { Stroke/TIA } \\
\mathrm{N}=404(8.1 \%)\end{array}$ & p Value \\
\hline \multicolumn{4}{|l|}{ Precipitating factors for $\mathrm{HF}$} \\
\hline Medications non-compliance (\%) & $878(19.1)$ & $86(21.3)$ & 0.28 \\
\hline Non-compliance with diet (\%) & $129(2.8)$ & $7(1.7)$ & 0.20 \\
\hline Salt retaining drugs (\%) & $26(0.6)$ & $0(0)$ & 0.13 \\
\hline Acute coronary syndrome (\%) & $1259(27.4)$ & $106(26.2)$ & 0.63 \\
\hline Uncontrolled hypertension (\%) & $374(8.1)$ & $36(8.9)$ & 0.58 \\
\hline Uncontrolled arrhythmia (\%) & $271(5.9)$ & $30(7.4)$ & 0.21 \\
\hline Anaemia (\%) & $138(3)$ & $16(4)$ & 0.28 \\
\hline Infection (\%) & $667(14.5)$ & $641(15.8)$ & 0.46 \\
\hline Unknown (\%) & $651(14.1)$ & $35(8.7)$ & 0.002 \\
\hline Worsening of renal failure & $197(4.3)$ & $24(5.9)$ & 0.12 \\
\hline NIV & 411 (8.9) & $62(15.3)$ & 0.001 \\
\hline Intubation/ventilation & $374(8.1)$ & $50(12.4)$ & 0.003 \\
\hline Inotropes & $695(15.1)$ & $88(21.8)$ & 0.001 \\
\hline IABP & $76(1.7)$ & $6(1.5)$ & 0.80 \\
\hline Acute dialysis/ultrafiltration & $117(2.5)$ & $18(4.5)$ & 0.02 \\
\hline VT/NF requiring therapy (\%) & $202(4.4)$ & $20(5.0)$ & 0.60 \\
\hline AF requiring therapy (\%) & $265(5.8)$ & $46(11.4)$ & 0.001 \\
\hline Major bleeding (\%) & $37(0.8)$ & $3(0.7)$ & 0.90 \\
\hline Blood transfusion (\%) & $217(4.7)$ & $37(9.2)$ & 0.001 \\
\hline In-hospital new stroke (\%) & $46(1)$ & $22(5.4)$ & 0.001 \\
\hline Systemic infection requiring antibiotics (\%) & 1067 (23.2) & $141(34.9)$ & 0.001 \\
\hline HHD & $725(15.8)$ & $77(19.1)$ & 0.08 \\
\hline IHD & 2424 (42.7) & $239(59.2)$ & 0.01 \\
\hline Primary VHD & $432(9.4)$ & $29(7.2)$ & 0.14 \\
\hline Viral myocarditis (\%) & $17(0.4)$ & $0(0.0)$ & 0.22 \\
\hline Cardiomyopathy (total) & $862(18.7)$ & $53(13.1)$ & 0.005 \\
\hline \multicolumn{4}{|l|}{ CM subtype } \\
\hline $\mathrm{HCM}$ & $19(0.4)$ & $3(0.7)$ & 0.34 \\
\hline Infiltrative CM & $12(0.3)$ & $1(0.2)$ & 0.96 \\
\hline Toxic CM & $36(0.8)$ & $3(0.7)$ & 0.93 \\
\hline Pregnancy-related CM & $63(1.4)$ & $2(0.5)$ & 0.14 \\
\hline Thyroid disease-related CM & $10(0.2)$ & $0(0)$ & 0.35 \\
\hline Familial CM & $9(0.2)$ & $0(0)$ & 0.37 \\
\hline Tachycardia-induced CM & $30(0.7)$ & $1(0.2)$ & 0.32 \\
\hline Idiopathic DCM & $605(13.1)$ & $37(9.2)$ & 0.02 \\
\hline Discharge home & 4104 (89.2) & $350(86.6)$ & 0.30 \\
\hline Transfer to another hospital & $80(1.7)$ & $8(2.0)$ & 0.30 \\
\hline Death & $279(6.1)$ & $34(8.4)$ & 0.06 \\
\hline Hospital stay (days) (mean $\pm S D)$ & $9 \pm 13$ & $11 \pm 14$ & 0.03 \\
\hline Alive (yes) & $3532(76.8)$ & $272(67.3)$ & 0.001 \\
\hline HF rehospitalisation (yes) (\%) & $989(28)$ & $86(31.6)$ & 0.20 \\
\hline \multicolumn{4}{|l|}{ Cardiac intervention needed } \\
\hline ICD & $33(0.7)$ & $1(0.2)$ & 0.27 \\
\hline CRTD/P & $12(0.2)$ & $2(0.5)$ & 0.33 \\
\hline $\mathrm{PCI} / \mathrm{CABG}$ & $358(10.1)$ & $22(8.1)$ & 0.28 \\
\hline
\end{tabular}

$\mathrm{AF}$, atrial fibrillation; CABG, coronary artery bypass grafting; CRTD, cardiac resynchronisation therapy, defibrillator; CRT, Cardiac resynchronization therapy; CM, cardiomyopathy; DCM, dilated cardiomyopathy; HCM, hypertrophic cardiomyopathy; HHD, hypertensive heart disease; IABP, intra-aortic balloon pump insertion; ICD, International Classification of Diseases; IHD, ischaemic heart disease; NIV, non-invasive ventilation; PCI, percutaneous coronary intervention; VF, ventricular fibrillation; VHD, valvular heart disease;

VT, ventricular tachycardia.

$\mathrm{HF}$ is a common disease and is a major risk factor for ischaemic stroke. Stroke-related morbidity and mortality are considerably higher in patients with $\mathrm{HF}$ compared with patients with stroke without $\mathrm{HF}^{22}$ Data on the prevalence and outcome of stroke in patients hospitalised for HF are very sparse and mainly conducted in the western world. To the best of our knowledge, this is the first study from the Middle East and the developing 
Table 6 Multivariate logistic regression analysis for in-hospital mortality

\begin{tabular}{|c|c|c|c|}
\hline Variable & Adjusted OR & $95 \% \mathrm{Cl}$ & p Value \\
\hline Age & 0.99 & 0.98 to 1.03 & 0.16 \\
\hline Male gender & 0.78 & 0.59 to 1.04 & 0.09 \\
\hline DM II & 1.06 & 0.79 to 1.41 & 0.70 \\
\hline CKD & 1.31 & 0.91 to 1.89 & 0.15 \\
\hline COPD/asthma & 0.64 & 0.37 to 1.10 & 0.11 \\
\hline STEMI & 2.25 & 1.57 to 3.23 & 0.001 \\
\hline LVEF $\geq 35 \%$ & 0.77 & 0.59 to 1.01 & 0.06 \\
\hline LVEF<35 & 1.30 & 0.99 to 1.70 & 0.06 \\
\hline VHD & 1.59 & 1.12 to 2.25 & 0.009 \\
\hline Stroke & 1.71 & 1.13 to 2.60 & 0.01 \\
\hline PVD & 1.52 & 0.89 to 2.62 & 0.13 \\
\hline \multicolumn{4}{|c|}{ Multivariate logistic regression analysis for 1 -year mortality } \\
\hline Age & 1.04 & 1.03 to 1.05 & 0.001 \\
\hline Male gender & 1.12 & 0.90 to 1.40 & 0.31 \\
\hline DM II & 1.18 & 0.95 to 1.46 & 0.13 \\
\hline CKD & 1.53 & 1.19 to 1.96 & 0.001 \\
\hline COPD/asthma & 1.22 & 0.91 to 1.65 & 0.19 \\
\hline STEMI & 0.89 & 0.62 to 1.29 & 0.55 \\
\hline LVEF $\geq 35$ & 0.74 & 0.60 to 0.91 & 0.005 \\
\hline LVEF $<35$ & 1.35 & 1.10 to 1.67 & 0.005 \\
\hline VHD & 1.46 & 1.10 to 1.93 & 0.009 \\
\hline Stroke & 1.34 & 0.98 to 1.84 & 0.07 \\
\hline PVD & 1.20 & 0.79 to 1.82 & 0.41 \\
\hline \multicolumn{4}{|c|}{ Risk factors for stroke in heart failure } \\
\hline Age & 1.02 & 1.01 to 1.03 & 0.001 \\
\hline Gender & 1.02 & 0.81 to 1.28 & 0.88 \\
\hline DM & 1.30 & 1.01 to 1.69 & 0.045 \\
\hline HTN & 2.10 & 1.50 to 2.80 & 0.001 \\
\hline Systolic BP & 1.0 & 0.99 to 1.01 & 0.51 \\
\hline Diastolic BP & 1.0 & 0.99 to 1.01 & 0.65 \\
\hline NYHA I & 0.64 & 0.19 to 2.14 & 0.33 \\
\hline NYHA II & 0.98 & 0.42 to 2.25 & 0.46 \\
\hline NYHA III & 1.24 & 0.55 to 2.80 & 0.95 \\
\hline NYHA IV & 1.24 & 0.54 to 2.81 & 0.61 \\
\hline Hyperlipidaemia & 1.30 & 1.02 to 1.65 & 0.03 \\
\hline CKD & 1.31 & 1.05 to 1.75 & 0.045 \\
\hline $\begin{array}{l}\text { Systolic LV } \\
\text { dysfunction }\end{array}$ & 1.08 & 0.85 to 1.37 & 0.52 \\
\hline Known CAD & 1.14 & 0.89 to 1.47 & 0.30 \\
\hline PVD (\%) & 2.97 & 2.06 to 4.12 & 0.001 \\
\hline $\mathrm{AF}$ & 2.20 & 1.67 to 2.89 & 0.001 \\
\hline Thyroid disease & 0.91 & 0.56 to 1.49 & 0.71 \\
\hline \multicolumn{4}{|c|}{$\begin{array}{l}\text { AF, atrial fibrillation; BP, blood pressure; CAD, coronary artery } \\
\text { disease; CKD, chronic kidney disease; COPD, chronic obstructive } \\
\text { pulmonary disease; DM, diabetes mellitus; HTN, hypertension; } \\
\text { LV, left ventricular; LVEF, left ventricular ejection fraction; NYHA, } \\
\text { New York Heart Association; PVD, peripheral vascular disease; } \\
\text { TIA, transient ischaemic attack; VHD, valvular heart disease. }\end{array}$} \\
\hline
\end{tabular}

world that explores this issue. We had previously reported a low prevalence of prior stroke in patients hospitalised with acute coronary syndrome (ACS) in the Middle East. ${ }^{23} 24$ On the other hand, this study reports a relatively higher prevalence of stroke in patients hospitalised with HF in seven Middle Eastern countries, suggesting that prior stroke is more prevalent among patients hospitalised with HF rather than ACS in this region.
Prevalence of stroke in patients with HF

The reported prevalence of stroke in patients with HF is variable because of the heterogeneous and limited number of published studies (table 8). ${ }^{25-31}$ The initial risk of ischaemic stroke during the first year after diagnosis of $\mathrm{HF}$ was reported to be $1.8 \%$; this compares with subsequent risk of nearly $5 \%$ at 5 years post-diagnosis. ${ }^{25}$ Data from the Framingham Study ${ }^{11}$ and a recent cohort study $^{12}$ indicated that the risk of ischaemic stroke is 2-3 times higher for patients with HF than it is for those without HF. According to epidemiological data, cohort studies and case series, $\approx 10 \%$ to $24 \%$ of all patients with stroke have HF, whereas $\mathrm{HF}$ is thought to be the likely cause of stroke in $\approx 9 \%$ of all patients. ${ }^{13-17}$ A meta-analysis including 15 clinical studies and 11 cohort studies published before $2006^{25}$ reported a stroke rate of $1.8 \%$ and $4.7 \%$ within 1 and 5 years, respectively. A recent report from the population-based, prospective Rotterdam Scan Study revealed that stroke risk is highest within 1 month after the diagnosis of HF that normalised within 6 months. ${ }^{32}$

This study complements previous reports by showing high prevalence of prior stroke among patients hospitalised with HF in the Middle East. Moreover, patients with a prior stroke had a higher risk of recurrent strokes during the index hospitalisation. This high prevalence may be due to associated risk factors for stroke such as severe $\mathrm{LV}$ systolic dysfunction (44.6\% had $\mathrm{EF}<35 \%)$ and $\mathrm{AF}(25 \%)$, even though there was a relatively high prevalence of associated risk factors, including $\mathrm{LV}$ dysfunction and $\mathrm{AF}$, while the use of anticoagulants and antiplatelet agents was low In this study, we reported the use of anticoagulants (20$22 \%)$ and antiplatelets (29-41\%) during hospitalisation at discharge, respectively, in patients with prior stroke; this rate may be much lower than has been reported in a EUROASPIRE III survey where antiplatelet drugs or oral anticoagulants were used by $87.2 \%$, of patients with stroke. ${ }^{33}$ This lower use of anticoagulants and antiplatelets in this study may have contributed to their increased risk of recurrent strokes and higher mortality rates.

\section{Risk factors for stroke in patients with HF and outcome}

Present facts concerning other risk factors for stroke in $\mathrm{HF}$ (apart from $\mathrm{AF}$ as the major factor) are primarily grounded on retrospective studies, cohort studies or post-hoc analyses of large clinical trials with significant inconsistencies. ${ }^{34}$ This study reported age, diabetes mellitus, hypertension, hyperlipidaemia, peripheral vascular disease and $\mathrm{AF}$ to be independently associated with recurrent stroke risk in patients with HF. The Olmsted County cohort demonstrated that prior stroke, advanced age and diabetes were relevant stroke risk factors in 630 patients with $\mathrm{HF}^{12}$ whereas a history of AF or hypertension did not reach statistical significance according to multivariable analysis. On the other side, a retrospective analysis of the prospective Survival and Ventricular Enlargement (SAVE) study also reported no significant impact of hypertension (and diabetes) in 2231 patients 
Table 7 Patient with HF with stroke/TIA as per care provider

\begin{tabular}{|c|c|c|c|}
\hline Variable & Cardiologist care 249 (61.6\%) & Internist care 155 (38.4\%) & p Value \\
\hline Acute new-onset HF (\%) & $83(33.3)$ & $43(27.7)$ & \\
\hline Acute decompensated chronic HF (\%) & $166(66.7)$ & $112(72.3)$ & 0.24 \\
\hline Age $($ mean $\pm S D)$ & $66 \pm 13$ & $67 \pm 12$ & 0.36 \\
\hline Male (\%) & $158(63.5)$ & $81(52.3)$ & 0.03 \\
\hline Previous admission for HF (\%) & $10(4)$ & $5(3.2)$ & 0.68 \\
\hline $\mathrm{AF}$ & $60(24.1)$ & $38(24.5)$ & 0.92 \\
\hline CKD/dialysis (\%) & $71(28.5)$ & $42(27.1)$ & 0.76 \\
\hline PVD & $44(17.7)$ & $17(11)$ & 0.07 \\
\hline CAD & $164(65.9)$ & $90(58.1)$ & 0.12 \\
\hline NYHA III & $106(42.6)$ & $82(52.9)$ & 0.04 \\
\hline NYHA IV & $83(33.3)$ & 49 (31.6) & 0.72 \\
\hline Clopidogrel & $120(48.2)$ & 47 (30.3) & 0.001 \\
\hline Oral anticoagulants (\%) & $60(24.1)$ & $31(20)$ & 0.34 \\
\hline ACE inhibitors & $129(51.8)$ & $59(38.1)$ & 0.007 \\
\hline ARBs & $43(17.3)$ & $34(21.9)$ & 0.25 \\
\hline Aldosterone antagonists & $88(35.3)$ & 47 (30.3) & 0.30 \\
\hline LVEF (mean $\pm S D)$ & $35 \pm 12.6$ & $39 \pm 14$ & 0.01 \\
\hline NIV & $37(14.9)$ & $25(16.1)$ & 0.73 \\
\hline Intubation/ventilation & 33 (13.3) & $17(11)$ & 0.50 \\
\hline Inotropes & $48(19.3)$ & $40(25.8)$ & 0.12 \\
\hline IABP & $5(2)$ & $1(0.6)$ & 0.27 \\
\hline Acute dialysis/ultrafiltration & $11(4.4)$ & $7(4.5)$ & 0.96 \\
\hline VT/VF requiring therapy (\%) & $14(5.6)$ & $6(3.9)$ & 0.43 \\
\hline $\mathrm{AF}$ requiring therapy $(\%)$ & $27(10.8)$ & $19(12.3)$ & 0.66 \\
\hline Major bleeding (\%) & $0(0)$ & $3(1.9)$ & 0.03 \\
\hline Blood transfusion (\%) & $16(6.4)$ & $21(13.5)$ & 0.02 \\
\hline In-hospital stroke (\%) & $5(2)$ & $17(11)$ & 0.001 \\
\hline Systemic infection requiring antibiotics (\%) & $69(27.7)$ & $72(46.5)$ & 0.001 \\
\hline Death in-hospital & $13(5.2)$ & $21(13.5)$ & 0.009 \\
\hline Hospital stay (days) (mean $\pm S D$ ) & $11 \pm 17$ & $10 \pm 10$ & 0.33 \\
\hline \multicolumn{4}{|l|}{ 1-year follow-up } \\
\hline Alive (yes) & $174(69.9)$ & $98(63.2)$ & 0.002 \\
\hline HF hospitalisation (yes) (\%) & $48(19.3)$ & $38(24.5)$ & 0.07 \\
\hline ICD & $0(0)$ & $1(0.6)$ & 0.20 \\
\hline CRTD & $2(0.8)$ & $0(0)$ & 0.26 \\
\hline $\mathrm{PCl} / \mathrm{CABG}$ & $13(7.5)$ & $9(9.2)$ & 0.62 \\
\hline
\end{tabular}

$\mathrm{AF}$, atrial fibrillation; ARBs, angiotensin receptors blockers; $C A B G$, coronary artery bypass grafting; CAD, coronary artery disease; CKD, chronic kidney disease; CRTD, Cardiac resynchronization therapy, defibrillator; HF, heart failure; IABP, intra-aortic balloon pump insertion; ICD, International Classification of Diseases; LVEF, left ventricular ejection fraction; NIV, non-invasive ventilation; NYHA, New York Heart Association; PCI, percutaneous coronary intervention; PVD, peripheral vascular disease; TIA, transient ischaemic attack, VF, ventricular fibrillation; VT, ventricular tachycardia.

with $\mathrm{HF}^{35}$ In contrast to these reports, the SCD-HeFTstudy revealed an $\mathrm{HR}$ of 1.9 (95\% CI 1.1 to 3.1 ) for stroke when hypertension was present at randomisation of 2144 patients with $\mathrm{HF}$ without $\mathrm{AF}^{9}$ In addition, a medical history of hypertension was associated with an increased risk of hospitalisation for stroke (HR 1.4; $95 \%$ CI 1.01 to 1.8 ) in 7788 patients with HF of the Digitalis Investigation Group trial. ${ }^{36}$ Furthermore, our result is compatible from the age point of view with the Olmsted County data that revealed a significant but modest association between stroke risk and advanced age (relative risk 1.04; $95 \%$ CI 1.02 to 1.06$).{ }^{12}$ An exploratory analysis of the SAVE study also showed similar results (relative risk of stroke 1.18 ; $95 \%$ CI 1.05 to 1.3 , for each increase of 5 years in age), ${ }^{9}$ while these results contradict the result of the Framingham Study that indicated that advanced age does not account for the increased risk of stroke in patients with $\mathrm{HF}^{3} 37$

This study has shown that patients with $\mathrm{HF}$ with stroke had higher in-hospital mortality with longer hospital stay and that they are less likely to stay alive at 1-year follow-up, probably explained by the multiple comorbidities in this patient population. Many retrospective database analyses have shown that stroke increases the disability and mortality of patients with HF through the alteration in neuropsychological status, like decreased attention and concentration, memory loss, diminished psychomotor speed and decreased executive function, and this ranged from approximately $25-80 \%$ of all patients with chronic $\mathrm{HF}^{38-41}$ 


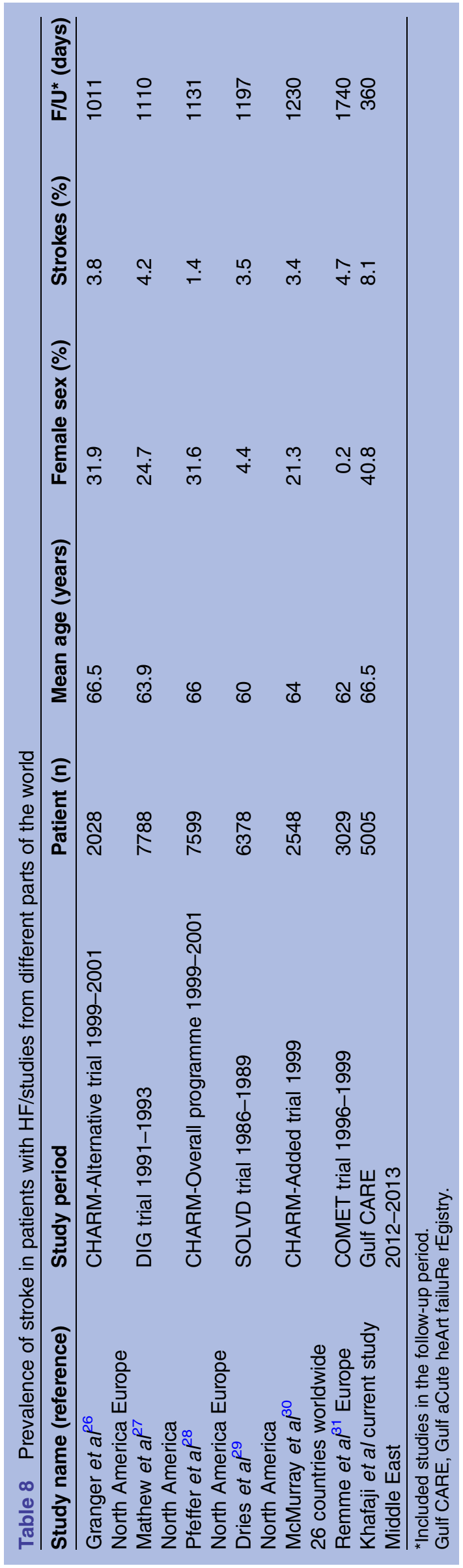

Cardiologist vs internist care of patients with HF with prior stroke

Cardiologists when compared with internists may provide more evidence-based therapies for the treatment of patients with $\mathrm{HF}^{42}{ }^{43}$ This is supported by this study, where patients under the care of cardiologists had a lower risk of recurrent in-hospital stroke, major bleeding, the need for blood transfusion and lower in-hospital and 1-year mortality rates when compared with patients managed by internists. This suggests that patients with prior stroke are a higher risk group which may benefit from specialised care. On the other hand, the observational nature of this study does not adjust for the possibility of selection bias in that patients with "lower" risk stroke were "preferentially" admitted under the care of cardiologists rather than internists.

\section{STUDY LIMITATIONS}

This study is a subanalysis of an observational study, which is like any observational study, where the possibility for unmeasured confounding variables exists. In addition, not all hospitals in each country participated; hence, the results cannot be generalised. This study did not record the cognitive status and the disability status in patients with stroke, which obviously have a major impact on morbidity and mortality and only 1-year mortality. Mortality rates at follow-up were only recorded at 1 year without the specification of the exact date of death of each patient, and hence the Kaplan-Meier curves could not have been done. Finally, no information was available regarding the cause of stroke (embolic vs thrombotic). Future studies need to overcome these limitations.

\section{CONCLUSION}

This observational study reports a high prevalence of prior stroke in Middle Eastern patients with HF. There was underuse of anticoagulation therapy in patients with stroke and HF with AF. Patients with stroke hospitalised with HF were more likely to be admitted under the care of internists rather than cardiologists, resulting in less use of evidencebased medications and worse outcome. Patients with stroke had a higher risk of in-hospital recurrent strokes and higher long-term mortality rates. History of stroke was an independent predictor of in-hospital and 1-year mortality rates in patients hospitalised with HF. Future studies are needed to evaluate whether aggressive evidence based therapies to this high-risk group will improve outcome.

\section{Author affiliations}

${ }^{1}$ Department of Cardiology, Saint Michael's Hospital, Toronto University, Canada

${ }^{2}$ Department of Cardiology, Royal Hospital, Muscat, Oman

${ }^{3}$ Biostatistics Section, Cardiovascular Research, Heart Hospital, Hamad Medical Corporation, Doha, Qatar

${ }^{4}$ Department of Cardiac Sciences, King Fahad Cardiac Center, King Saud University, Riyadh, Saudi Arabia

${ }^{5}$ Adult Cardiology, Heart Hospital, Hamad Medical Corporation, Doha, Qatar ${ }^{6}$ Department of Cardiology, Sheikh Khalifa Medical City, Abu Dhabi, UAE

${ }^{7}$ Department of Cardiology, Sabah Al-Ahmed Cardiac Center, Kuwait 
${ }^{8}$ Department of Cardiology, Adan Hospital, Kuwait, Kuwait

${ }^{9}$ Department of Cardiology, Dubai hospital, Dubai, UAE

${ }^{10}$ Department of Cardiology, Mohammed Bin Khalifa Cardiac Center,

Manamah, Bahrain

${ }^{11}$ Department of Cardiology, Faculty of Medicine, Sana'a University, Sana'a, Yemen

${ }^{12}$ Department of Cardiology, Prince Salman Heart Center, King Fahad Medical City, Saudi Arabia

${ }^{13}$ Department or Cardiology, Tanta University, Tanta, Egypt

${ }^{14}$ Qatar Cardiovascular Research Center and Adult Cardiology, Heart Hospital, Hamad Medical Corporation, Doha, Qatar

Acknowledgements We thank Hani Altaradi and Kazi Nur Asfina for research coordination.

Contributors KS, KFA, NA, AA-A, MA-J, BB, WA, MR, NB, HA, AA-M, HA, AE, $P P$ and JA were involved in the design of the Gulf CARE registry and patient enrolment and ensuring quality control of the study. RS carried out the statistical analyses. All authors contributed to the drafting of the article and approved the final version of the manuscript.

Funding Gulf CARE is an investigator-initiated study conducted under the auspices of the Gulf Heart Association and funded by Servier, Paris, France; and (for centres in Saudi Arabia) by the Saudi Heart Association.

Competing interests None.

Provenance and peer review Not commissioned; externally peer reviewed.

Data sharing statement No additional data are available.

Open Access This is an Open Access article distributed in accordance with the Creative Commons Attribution Non Commercial (CC BY-NC 4.0) license, which permits others to distribute, remix, adapt, build upon this work noncommercially, and license their derivative works on different terms, provided the original work is properly cited and the use is non-commercial. See: http:// creativecommons.org/licenses/by-nc/4.0/

\section{REFERENCES}

1. Häusler KG, Laufs U, Endres M. Neurological aspects of chronic heart failure. Nervenarzt 2011;82:733-42.

2. Pullicino PM, Halperin JL, Thompson JL. Stroke in patients with heart failure and reduced left ventricular ejection fraction. Neurology 2000;54:288-94.

3. Wolf PA, Abbott RD, Kannel WB. Atrial fibrillation is an independent risk factor for stroke: the Framingham Study. Stroke 1991;22:983-8.

4. Caldwell JC, Mamas MA, Neyses L, et al. What are the thromboembolic risks of heart failure combined with chronic or paroxysmal AF? J Card Fail 2010;16:340-7.

5. Jug B, Vene N, Salobir BG, et al. Procoagulant state in heart failure with preserved left ventricular ejection fraction. Int Heart J 2009;50:591-600.

6. Georgiadis D, Sievert M, Cencetti S, et al. Cerebrovascular reactivity is impaired in patients with cardiac failure. Eur Heart $J$ 2000;21:407-13.

7. Lip GY, Gibbs CR. Does heart failure confer a hypercoagulable state? Virchow's triad revisited. J Am Coll Cardiol 1999;33:1424-6.

8. Pullicino PM, McClure LA, Wadley VG, et al. Blood pressure and stroke in heart failure in the REasons for Geographic And Racial Differences in Stroke (REGARDS) study. Stroke 2009;40:3706-10.

9. Freudenberger RS, Hellkamp AS, Halperin JL, et al. Risk factors for thromboembolism in the SCD-Heft Study. Circulation 2007;115:2637-41.

10. Pullicino P, Mifsud V, Wong E, et al. Hypoperfusion-related cerebral ischemia and cardiac left ventricular systolic dysfunction. J Stroke Cerebrovasc Dis 2001;10:178-82.

11. Kannel WB, Wolf PA, Verter J. Manifestations of coronary disease predisposing to stroke: the Framingham Study. J Am Med Assoc 1983;250:2942-6.

12. Witt BJ, Brown RD Jr, Jacobsen SJ, et al. Ischemic stroke after heart failure: a community-based study. Am Heart J 2006;152:102-9.

13. Appelros P, Nydevik I, Viitanen M. Poor outcome after first-ever stroke: predictors for death, dependency, and recurrent stroke within the first year. Stroke 2003;34:122-6.

14. Divani AA, Vazquez G, Asadollahi M, et al. Nationwide frequency and association of heart failure on stroke outcomes in the United States. J Card Fail 2009;15:11-16.
15. Hays AG, Sacco RL, Rundek T, et al. Left ventricular systolic dysfunction and the risk of ischemic stroke in a multiethnic population. Stroke 2006;37:1715-19.

16. Ois A, Gomis M, Cuadrado-Godia E, et al. Heart failure in acute ischemic stroke. J Neurol 2008;255:385-9.

17. Pullicino $\mathrm{P}, \mathrm{Homma} \mathrm{S}$. Stroke in heart failure: atrial fibrillation revisited? J Stroke Cerebrovasc Dis 2010;19:1-2.

18. Sulaiman KJ, Panduranga P, Al-Zakwani I, et al. Rationale, Design, Methodology and Hospital Characteristics of the First Gulf Acute Heart Failure Registry (Gulf CARE). Heart Views 2014;15:6-12.

19. Radford MJ, Arnold JM, Bennett SJ, et al; American College of Cardiology; American Heart Association Task Force on Clinical Data Standards; American College of Chest Physicians, et al;. ACC/AHA key data elements and definitions for measuring the clinical management and outcomes of patients with chronic heart failure: a report of the American College of Cardiology/American Heart Association Task Force on Clinical Data Standards (Writing Committee to Develop Heart Failure Clinical Data Standards): developed in collaboration with the American College of Chest Physicians and the International Society for Heart and Lung Transplantation: endorsed by the Heart Failure Society of America. Circulation 2005;112:1888-916.

20. Dickstein K, Cohen-Solal A, Filippatos G, et al. ESC guidelines for the diagnosis and treatment of acute and chronic heart failure 2008: the Task Force for the Diagnosis and Treatment of Acute and Chronic Heart Failures 2008 of the European Society of Cardiology. Developed in collaboration with the Heart Failure Association of the ESC (HFA) and endorsed by the European Society of Intensive Care Medicine (ECSIM). Eur Heart J 2008;29:2388-442.

21. Hosmer DW Jr, Lemeshow S, Sturdivant RX. Introduction to the Logistic Regression Model, in Applied Logistic Regression. 3rd edn. Hoboken, NJ, USA: John Wiley \& Sons, Inc., 2013.

22. Haeusler KG, Laufs U, Endres M. Chronic heart failure and ischemic stroke. Stroke 2011:42:2977-82.

23. Al Suwaidi J, Al Habib K, Asaad N, et al. Immediate and one-year outcome of patients presenting with acute coronary syndrome complicated by stroke: findings from the 2nd Gulf Registry of Acute Coronary Events (Gulf RACE-2). BMC Cardiovasc Disord 2012;12:64.

24. Albaker O, Zubaid M, Alsheikh-Ali AA, et al. Gulf RACE Investigators. Early stroke following acute myocardial infarction: incidence, predictors and outcome in six Middle-Eastern countries. Cerebrovasc Dis 2011;32:471-82.

25. Witt BJ, Gami AS, Ballman KV, et al. The incidence of ischemic stroke in chronic heart failure: a meta-analysis. J Card Fail 2007;13:489-96.

26. Granger C, McMurray J, Yusuf S, et al. Effects of candesartan in patients with chronic heart failure and reduced left-ventricular systolic function intolerant to angiotensin-converting-enzyme inhibitors: the CHARM-Alternative trial. Lancet 2003;362:772-6.

27. Mathew J, Hunsberger S, Fleg J, et al. Incidence, predictive factors, and prognostic significance of supraventricular tachyarrhythmias in congestive heart failure. Chest 2000;118:914-22.

28. Pfeffer M, Swedberg K, Granger C, et al. Effects of candesartan on mortality and morbidity in patients with chronic heart failure: the CHARM-Overall programme. Lancet 2003;362:759--66.

29. Dries DL, Rosenberg YD, Waclawiw MA, et al. Ejection fraction and risk of thromboembolic events in patients with systolic dysfunction and sinus rhythm: evidence for gender differences in the studies of left ventricular dysfunction trials [erratum appears in $\mathrm{J}$ Am Coll Cardiol 1998;32:555]. J Am Coll Cardiol 1997;29:1074--80.

30. McMurray J, Ostergren J, Swedberg K, et al. Effects of candesartan in patients with chronic heart failure and reduced left-ventricular systolic function taking angiotensin-converting-enzyme inhibitors: the CHARM-Added trial. Lancet 2003;362:767-71.

31. Remme W, Cleland J, DiLenarda A, et al. Carvedilol better protects against vascular events than metoprolol in heart failure: results from COMET. J Am Coll Cardiol 2004;43(Suppl 1):A205-6.

32. Alberts VP, Bos MJ, Koudstaal PJ, et al. Heart failure and the risk of stroke: the Rotterdam Study. Eur J Epidemiol 2010;25:807-12.

33. Heuschmann PU, Kircher J, Nowe T, et al. Control of main risk factors after ischaemic stroke across Europe: data from the stroke-specific module of the EUROASPIRE III survey. Eur J Prev Cardiol 2014. doi:10.1177/2047487314546825 [Epub ahead of print 19 Aug 2014].

34. Pullicino P, Thompson JL, Mohr JP, et al. Oral anticoagulation in patients with cardiomyopathy or heart failure in sinus rhythm. Cerebrovasc Dis 2008;26:322-7.

35. Loh E, Sutton MS, Wun CC, et al. Ventricular dysfunction and the risk of stroke after myocardial infarction. $N$ Engl J Med 1997;336:251-7. 
36. Filippatos GS, Adamopoulos C, Sui X, et al. A propensity-matched study of hypertension and increased stroke-related hospitalization in chronic heart failure. Am J Cardiol 2008;101:1772-6.

37. Wolf PA, Abbott RD, Kannel WB. Atrial fibrillation: a major contributor to stroke in the elderly: the Framingham Study. Arch Intern Med 1987;147:1561-4.

38. Heckman GA, Patterson CJ, Demers C, et al. Heart failure and cognitive impairment: challenges and opportunities. Clin Interv Aging 2007;2:209-18.

39. Debette S, Bauters C, Leys D, et al. Prevalence and determinants of cognitive impairment in chronic heart failure patients. Congest Heart Fail 2007;13:205-8.
40. Pressler SJ. Cognitive functioning and chronic heart failure: a review of the literature. J Cardiovasc Nurs 2008;23:239-49.

41. Zuccalà G, Pedone $\mathrm{C}$, Cesari $\mathrm{M}$, et al. The effects of cognitive impairment on mortality among hospitalized patients with heart failure. Am J Med 2003;115:97-103.

42. Philbin EF, Jenkins PL. Differences between patients with heart failure treated by cardiologists, internists, family physicians, and other physicians: analysis of a large, statewide database. Am Heart J 2000;139:491-6.

43. Jong P, Gong Y, Liu PP, et al. Care and outcomes of patients newly hospitalized for heart failure in the community treated by cardiologists compared with other specialists. Circulation 2003;108:184-91. 